\title{
Radiotherapy planning parameters correlate with changes in the peripheral immune status of patients undergoing curative radiotherapy for localized prostate cancer
}

\author{
Elgin Hoffmann ${ }^{1} \cdot$ Frank Paulsen $^{1} \cdot$ Philipp Schaedle ${ }^{1,2,4} \cdot$ Daniel Zips $^{1,3} \cdot$ Cihan Gani $^{1,3} \cdot$ Hans-Georg Rammensee $^{2,3,5}$. \\ Cécile Gouttefangeas ${ }^{2,3,5} \cdot$ Franziska Eckert $^{1,3}$ (1)
}

Received: 3 May 2021 / Accepted: 28 June 2021 / Published online: 16 July 2021

(c) The Author(s) 2021

\begin{abstract}
Purpose The influence of radiotherapy on patient immune cell subsets has been established by several groups. Following a previously published analysis of immune changes during and after curative radiotherapy for prostate cancer, this analysis focused on describing correlations of changes of immune cell subsets with radiation treatment parameters.

Patients and methods For 13 patients treated in a prospective trial with radiotherapy to the prostate region (primary analysis) and five patients treated with radiotherapy to prostate and pelvic nodal regions (exploratory analysis), already published immune monitoring data were correlated with clinical data as well as radiation planning parameters such as clinical target volume (CTV) and volumes receiving $20 \mathrm{~Gy}$ (V20) for newly contoured volumes of pelvic blood vessels and bone marrow. Results Most significant changes among immune cell subsets were observed at the end of radiotherapy. In contrast, correlations of age and $\mathrm{CD}^{+}$subsets (effector and memory cells) were observed early during and 3 months after radiotherapy. Ratios of T cells and T cell proliferation compared to baseline correlated with CTV. Early changes in regulatory T cells (Treg cells) and $\mathrm{CD} 8^{+}$effector $\mathrm{T}$ cells correlated with V20 of blood vessels and bone volumes.

Conclusions Patient age as well as radiotherapy planning parameters correlated with immune changes during radiotherapy. Larger irradiated volumes seem to correlate with early suppression of anti-cancer immunity. For immune cell analysis during normofractionated radiotherapy and correlations with treatment planning parameters, different time points should be looked at in future projects.
\end{abstract}

Trial registration number: NCT01376674, 20.06.2011

Keywords Prostate cancer $\cdot$ Localized $\cdot$ IMRT $\cdot$ DVH $\cdot$ Immune status $\cdot$ T cells

Franziska Eckert

franziska.eckert@med.uni-tuebingen.de

1 Department of Radiation Oncology, University Hospital Tuebingen, Eberhard-Karls-University Tuebingen, Hoppe-Seyler-Str. 3, 72076 Tuebingen, Germany

2 Interfaculty Institute for Cell Biology, Department of Immunology, Eberhard-Karls-University Tuebingen, Auf der Morgenstelle 15, 72076 Tuebingen, Germany

3 German Cancer Consortium (DKTK), German Cancer Research Center (DKFZ) Partner Site Tuebingen, Tuebingen, Germany

4 Present Address: Department for Internal Medicine I, Marienhospital Stuttgart, Boeheimstr. 37, 70199 Stuttgart, Germany

5 Cluster of Excellence iFIT (EXC2180) 'Image-Guided and Functionally Instructed Tumor Therapies', University of Tuebingen, Tuebingen, Germany

\section{Introduction}

Radiotherapy, mostly in combination with androgen deprivation therapy (ADT), is one of the curative options for localized prostate adenocarcinoma. Depending on the risk classification, tumor control rates of $>85 \%,>75 \%$ and $>55 \%$ for low, intermediate and high-risk prostate cancer patients can be achieved after radiotherapy with $74 \mathrm{~Gy}$ [1]. With imageguidance and conformal radiotherapy planning, higher radiation doses have become achievable without excessive toxicity [2].

Although not established in clinical routine for the treatment of localized prostate cancer [3], immunotherapeutical approaches have become a major player in oncology in general [4], including for metastatic prostate cancer [5]. Several clinical trials have been published and are ongoing $[6,7]$. 
Combining immunotherapy with radiotherapy has become a major field of investigation [8-10]. However, there are a lot of uncertainties about the specifics of radiotherapy (dose, fractionation, timing) to be best combined with immunotherapy. Sequencing of different therapy modalities, as well as fractionation and dosing of radiotherapy, seems to play a major role for synergistic effects [11]. For the combination with PD1 / PD-L1 blockade, simultaneous application has been described as favorable in preclinical models [12]. In contrast, the effect of adoptive $T$ cell transfer was most pronounced when given at late time points after radiotherapy [13]. There is a strong rationale for hypofractionated irradiation in combination with CTLA4 blockade [14]. Optimal sequencing, fractionation and total doses will probably vary for the immunotherapy modality applied $[15,16]$.

Immune biomarker assessment in patients undergoing cancer therapy should generate helpful data for determination of which time points in standard cancer therapies might be best for combination with different immunotherapy approaches and which clinical settings and treatment schedules might be most promising. Multiparameter flow cytometry is the method of choice for analyzing immune cell subsets in peripheral blood over time [17, 18]. Moreover, analysis of changes in subsets of immune cells during standard cancer treatments might help in finding the optimal time point and scheduling for future treatment options. Examples for informative reports are studies which observed that definitive radiochemotherapy for cervical cancer led to profound immunosuppression which might limit the advantage of combined approaches with immunotherapy [19]. For patients undergoing surgery for lung cancer, several blood immune cell subsets showed prognostic significance [20]. Our own data, on which this report is based, have shown significant changes in peripheral immune cell subsets in prostate cancer patients undergoing definitive radiotherapy ( \pm androgen deprivation), not all of which had subsided three months after end of treatment [21].

The relationship between radiation dose to the pelvic bone marrow and hematologic toxicity, mostly leukopenia and lymphopenia, has been described for radiochemotherapy of cervical [22] and anal cancers [23]. For radiotherapy of prostate cancer patients, long-lasting lymphopenia was observed, especially for prescribed higher doses to nodal volumes and for older patients [24]. Lymphopenia was also related to the radiation dose to pelvic bone marrow as described by Sini et al. [25].

Cell subsets analyzed in this study comprise general categories such as T cells and B cells, but also functionally characterized subsets. Effector T cells are cells directly involved in anti-cancer immune responses [26]. Memory T cells play a crucial role in long-lasting immunity and protection against relapses of cancer after curative treatment [27]. Treg cells are the subset responsible for ending overwhelming immune responses after infections. In cancer immunology, they are well known for negatively impacting anti-cancer immunity [28].

The aim of this study was to relate our already published immunomonitoring data for prostate cancer patients undergoing definitive radiotherapy [21] with radiation planning parameters (target volume, treated volume of immuneassociated structures such as pelvic bone marrow and large pelvic blood vessels). With the in-depth immunomonitoring data acquired during this study, a detailed description of immunologic changes is possible.

\section{Patients and methods}

\section{Patients and treatment}

In a prospective study, peripheral blood mononuclear cells (PBMCs) from 18 of 63 initially screened patients were collected during and after curative radiotherapy with $(n=16)$ or without $(\mathrm{n}=2)$ androgen deprivation therapy in the years 2011 and 2012 . The study was approved by the local ethics committee (project number 402/2010BO2) and registered at www.clinicaltrials.gov (NCT01376674). The results describing longitudinal assessments of various immune cell subsets were published in 2018 [21]. Patients were treated with $70-78$ Gy in 35 to 39 fractions to the prostate with base of seminal vesicles in case of stage T3a or less or to the prostate with entire seminal vesicles in case of T3b stage, respectively, according to institutional standards at that time. Of the 18 patients included in this analysis (median age of 75 years, range $68-82$ years), five were also treated with 50.4 Gy to the pelvic nodal regions, offered to patients with high-risk prostate cancer. Radiotherapy was planned based on three planning CTs using a coverage probability approach as published previously $[29,30]$. Due to large differences in dose levels to pelvic areas for patients treated with elective nodal irradiation, the primary analysis was focused on the patient cohort undergoing radiotherapy to prostate with / without seminal vesicles only. The five patients treated with elective nodal irradiation were analyzed separately in an exploratory analysis.

\section{Immune cell subsets}

Peripheral immune cell subsets were characterized by multiparameter flow cytometry as described in detail previously [21]. Blood samples were obtained before start of treatment (time point A), twice during therapy at 1-month intervals (time points B, C) and three months after the end of treatment at a follow-up visit (time point D). Sample size for cell sub-populations analyzed at each time point is specified in Suppl. Table 1. PBMCs were isolated by density gradient 
centrifugation and frozen until use. All PBMCs from each patient were analyzed in the same experiment. $\mathrm{T}$ cells were defined as $\mathrm{CD} 3{ }^{+} \mathrm{CD} 19^{-}$lymphocytes and further characterized as $\mathrm{CD}^{+}{ }^{+}$or $\mathrm{CD}^{+}$. Regulatory $\mathrm{T}$ cells were identified as $\mathrm{CD} 4^{+} \mathrm{CD} 25^{+} \mathrm{FoxP}^{+}{ }^{+} \mathrm{T}$ cells, $\mathrm{B}$ cells as $\mathrm{CD} 3^{-} \mathrm{CD} 19^{+}$and natural killer cells (NK) as $\mathrm{CD}^{-} \mathrm{CD} 19^{-}$lymphocytes. Proliferation status $\left(\mathrm{Ki}_{67}{ }^{+}\right)$was assessed for all of these subsets. Naïve T cells were defined as $\mathrm{CD} 45 \mathrm{RA}^{+} \mathrm{CD} 28^{+}$, effector

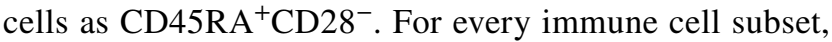
intra-individual ratios of the percentages at timepoints $\mathrm{B}, \mathrm{C}$ and $\mathrm{D}$ were calculated referring to baseline (time point $\mathrm{A}$ ).

\section{Analysis of radiotherapy planning parameters}

All radiation treatment plans were calculated with Hyperion ${ }^{\circledR}$, a Monte-Carlo-based treatment planning system using an EUD (equivalent uniform dose)-based optimization concept. All treatments were planned as static IMRT using 8 gantry angles in 17 cases, 5 gantry angles in one case. All radiation treatment plans were reviewed, and dose and volume parameters were recorded for clinical target volume (CTV) and planning target volume (PTV).

\section{Contouring of pelvic bone marrow and vessels}

For all patients, additional volumes with a possible relationship to immune parameters were contoured, namely bone marrow, pelvic and iliac lymph nodes and large vessels. Pelvic bone marrow, as well as pelvic bones, was contoured as iliac left and right, lumbosacral and lower pelvic volumes for two patients. Dose parameters were compared for bone marrow and bone structures, respectively. Further analysis was performed with bone structures. The hull was termed pelvic bone marrow union (PBM union) following the contouring as suggested by Li et al. [31]. In addition, large pelvic blood vessels were contoured, with craniocaudal borders defined by the bone marrow contour borders, including aorta and external iliac and inguinal arteries and veins, the hull being termed vessels. Dose-volume histograms (DVH) for the original, clinically used treatment plans, were extracted from the planning system, and percentage of volume receiving $\mathrm{X}$ Gy or more (VX; $\mathrm{X}=10,20,30,40$ and 50) were recorded for every volume. For further analysis, V20 values were used for contoured blood vessels as well as total pelvic bone marrow (PBM). V20 was chosen as it was the highest dose level with values $>0 \mathrm{~cm}^{3}$ in all patients. Fraction dose for V20 in 35-37 fractions is $0.54-0.57 \mathrm{~Gy}$ and thus a range with a predicted biological effect, corresponding to the dose with a $90 \%$ survival fraction of lymphocytes [32]. For PBM union correlation of V10, V30, V40 and V50 were analyzed additionally. For large blood vessels analysis of V10 and V30 was performed as well.

\section{Statistical analysis}

The statistical analysis was done with GraphPad 8.4.0 (GraphPad, San Diego, CA, the USA) and SPSS24 (SPSS Inc., Chicago, IL, the USA). Correlations of linear parameters were characterized by the Pearson correlation coefficient (r). Moderate and strong correlations were defined by Pearson correlation coefficients of $0.4-0.7$ and $>0.7$, respectively. Means were compared by student's t test if the assumptions for the test were met and Bonferroni corrected in case of multiple testing. $P$ values were considered significant if $<0.05$, with Bonferroni correction $\mathrm{zp}<0.05$ $(\mathrm{z}=$ number of comparisons). Means are given \pm standard error of the mean.

\section{Results}

\section{Immune cell ratios compared to baseline}

In order to correlate immune cell changes during radiotherapy with radiation planning parameters, intra-individual ratios of immune cell subsets were calculated for each time point during or post-radiotherapy (time point B (4 weeks into radiotherapy), time point $\mathrm{C}$ (end of treatment) and time point $\mathrm{D}$ (first follow-up after three months)) compared to before therapy (time point A) as shown in Fig. 1. The values used for ratio calculation, which were $\%$ of the cell subsets of interest, have been reported in a previous publication [21]. Significant early changes at time point B were limited to increased proliferation of most tested cell subsets except for Tregs (Fig. 1, upper panel). Percentages of cell numbers did not show significant alterations. Most significant changes in immune cell subsets, as well as in proliferation, were observed comparing time point $\mathrm{C}$ to time point $\mathrm{A}$ (Fig. 1, middle panel). $\mathrm{T}$ cells and B cells were significantly decreased, while NK and Treg cell frequencies were significantly higher compared to baseline. In the $\mathrm{CD}^{+}{ }^{+}$subset, effector cells were more prominent, and inversely naïve cells were decreased. All cell subsets showed a significantly increased proliferation. Three months after end of the radiotherapy, most immune cell changes had recovered with the exception of low naïve $\mathrm{CD}^{+}$and $\mathrm{CD}^{+}{ }^{+} \mathrm{T}$ cells, and an enhanced proliferation rate of whole $\mathrm{CD} 8^{+} \mathrm{T}$ cells (Fig. 1, time point $\mathrm{D}$ to time point A, lower panel). As two patients were treated without ADT, we plotted the data of these patients separately in comparison to the eleven patients with bimodal treatment. The small number of patients did not allow for statistical 
Fig. 1 To compare immune cell changes with radiation planning parameters, the ratio of immune cell subsets and proliferative fractions within each of these cell subsets is displayed for time point $\mathrm{B}$ (4 weeks into radiotherapy), time point $\mathrm{C}$ (end of radiotherapy) and time point $\mathrm{D}$ (3 months after end of radiotherapy) compared to baseline levels (time point A). Significant changes have been observed for proliferation of most cell subsets at time point $\mathrm{B}$, most subsets at time point $\mathrm{C}$, and only a few long-lasting effects at time point $\mathrm{D}$. The red line indicates a ratio of 1 and thus no change compared to baseline frequencies. * indicates significant changes with one sample t-test compared to a ratio of 1
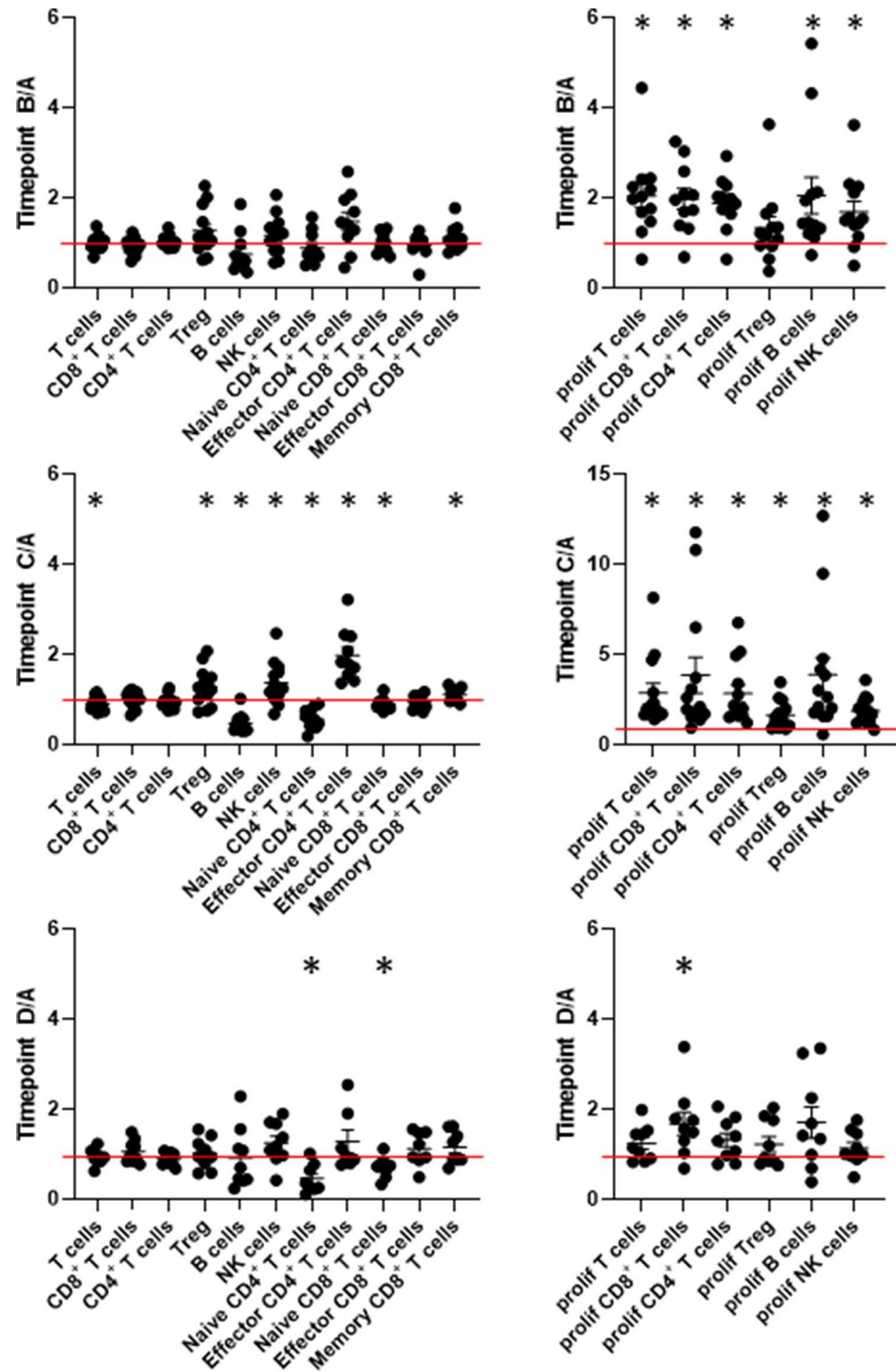
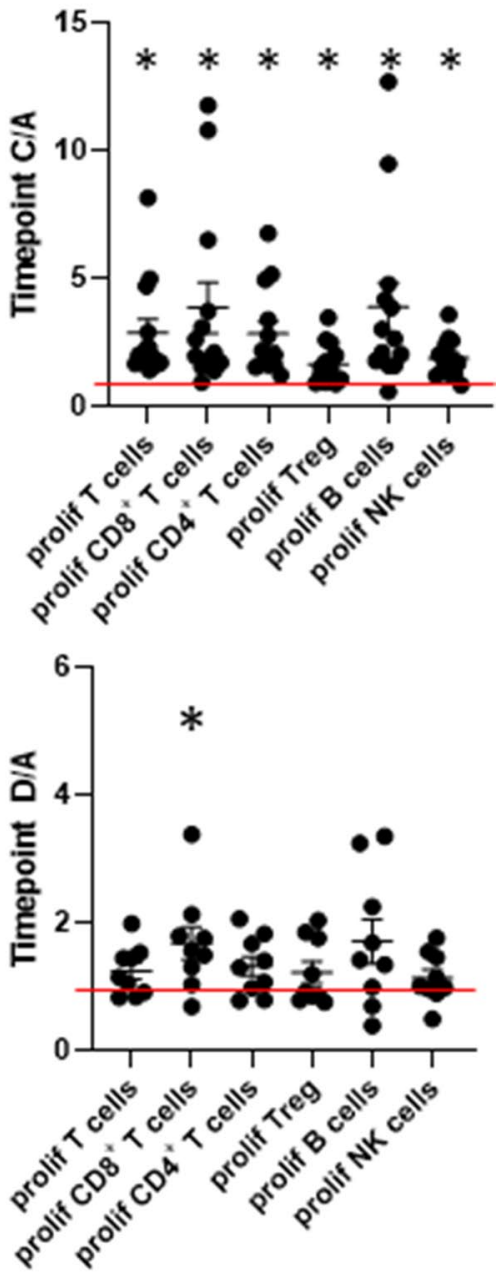

analysis, but no marked differences were detected (data not shown). Thus, for all further analyses, these two patients were included.

\section{Immune cell ratios and patient age}

Considering the impact of aging on the immune system, we next asked whether the changes in immune cell subsets were impacted by the age of the patients. Ratios at the earliest time during radiotherapy (time point $\mathrm{B}$ ) showed moderate 

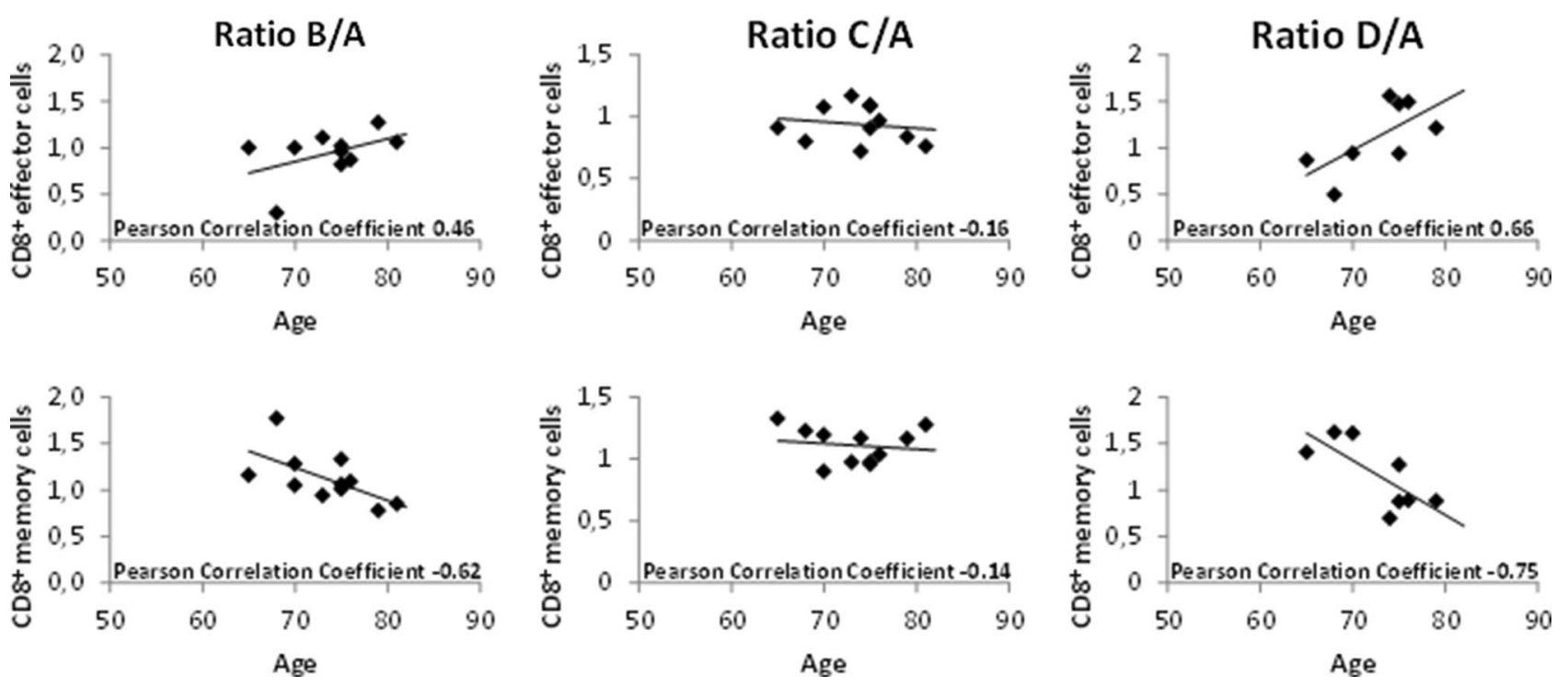

Fig. 2 Correlation of patient age at time of radiotherapy and $\mathrm{CD} 8^{+}$ effector cells as well as $\mathrm{CD} 8^{+}$memory cells is shown for all time points in the subgroup of patients treated with radiotherapy to the

prostate. Patient age correlated positively with $\mathrm{CD} 8^{+}$effector cells and negatively with $\mathrm{CD} 8^{+}$memory cells at early and late time points (B/A and D/A, respectively)

or strong correlations with patient age at start of treatment. Among other correlations (for details, refer to Suppl. Table 2, listing all Pearson correlation coefficients), a positive correlation for $\mathrm{CD} 8^{+}$effector cells and a negative correlation for $\mathrm{CD}^{+}$memory cells were observed (ratio B/A, Fig. 2 left panel). These were lost at a later time point during therapy (ratio C/A, Fig. 2. middle panel), but again observed during the recovery phase 3 months after treatment (ratio D/A, Fig. 2, right panel). Percentage of $\mathrm{CD} 8^{+}$effector cells and $\mathrm{CD}^{+}$memory cells at time points $\mathrm{A}, \mathrm{B}, \mathrm{C}$ and $\mathrm{D}$ considered separately did not correlate with patient age (data not shown). Only one moderate positive correlation was found at the end of radiotherapy for $\mathrm{CD} 4{ }^{+}$effector cells and patient age (data not shown). These results were not confirmed in the exploratory cohort (data not shown).

\section{Contouring of lymphocyte rich structures}

In order to compare intra-individual ratios of immune cell subsets with radiation planning parameters beyond the size of target volumes, lymphocyte-rich structures were contoured for every treatment plan, and DVH parameters of the clinically applied radiation plans were extracted. The pelvic bones were contoured in analogy to $\mathrm{Li}$ et al. [31]. For two patients, pelvic bones as well as pelvic bone marrow were contoured in order to compare DVH parameters for both volumes. DVH parameters for iliac bone marrow, lower pelvic bone marrow and lumbosacral bone marrow were compared (V10, V20, V30, V40, V50) to corresponding data for the whole bones including calcified regions, and no significant difference was detected (comparison of dose parameters for 6 volumes, data not shown). As a consequence, for all other patients $(n=16)$, only pelvic bones as a whole were contoured. The respective contoured volumes for all patients were $266.1 \pm 8.1 \mathrm{~cm}^{3}, 448.3 \pm 18.1 \mathrm{~cm}^{3}$, $723.3 \pm 22.7 \mathrm{~cm}^{3}$ for iliac bone marrow, lumbosacral bone marrow and lower pelvic bone marrow, respectively. The analysis was performed with V20 of the pelvic bone marrow (hull of iliac bone marrow, lower pelvic bone marrow and lumbosacral bone marrow, PBM union) with a mean volume of $1708.1 \pm 46.2 \mathrm{~cm}^{3}$. In order to compare radiation doses to the blood vessels to immune cell subsets in the peripheral blood, large pelvic blood vessels were contoured as well. These include the external iliac vessels (arteries and veins) starting at the bifurcation, reaching to inguinal and femoral region. Contoured volume was $193.1 \pm 8.3 \mathrm{~cm}^{3}$. For these volumes analysis was also performed with V20 values. Contoured volumes and CTV, rectum, bladder and vessels are shown for one patient in Fig. 3.

Dose-volume histograms of the five patients treated to pelvic node irradiation show a marked difference in dose levels to PBM union and vessels compared to patients treated to prostate volumes only (Fig. 4, upper panels). Thus, pooling all patients for analysis with immune parameters is not reasonable. Primary analysis was limited to the patients, who received radiotherapy to the prostate only. The five patients treated with radiotherapy to elective nodal regions were used for an exploratory analysis. 
Fig. 3 Volumes contoured in addition to standard radiotherapy target volume (turquoise) and organs at risk such as bladder (yellow) and rectum (brown) are pelvic bone marrow (PBM union) comprising iliac bone marrow (red), lumbosacral spine (yellow) and lower pelvic bone marrow (light blue) as well as arteries (orange) and veins (dark blue) combined as vessels are shown

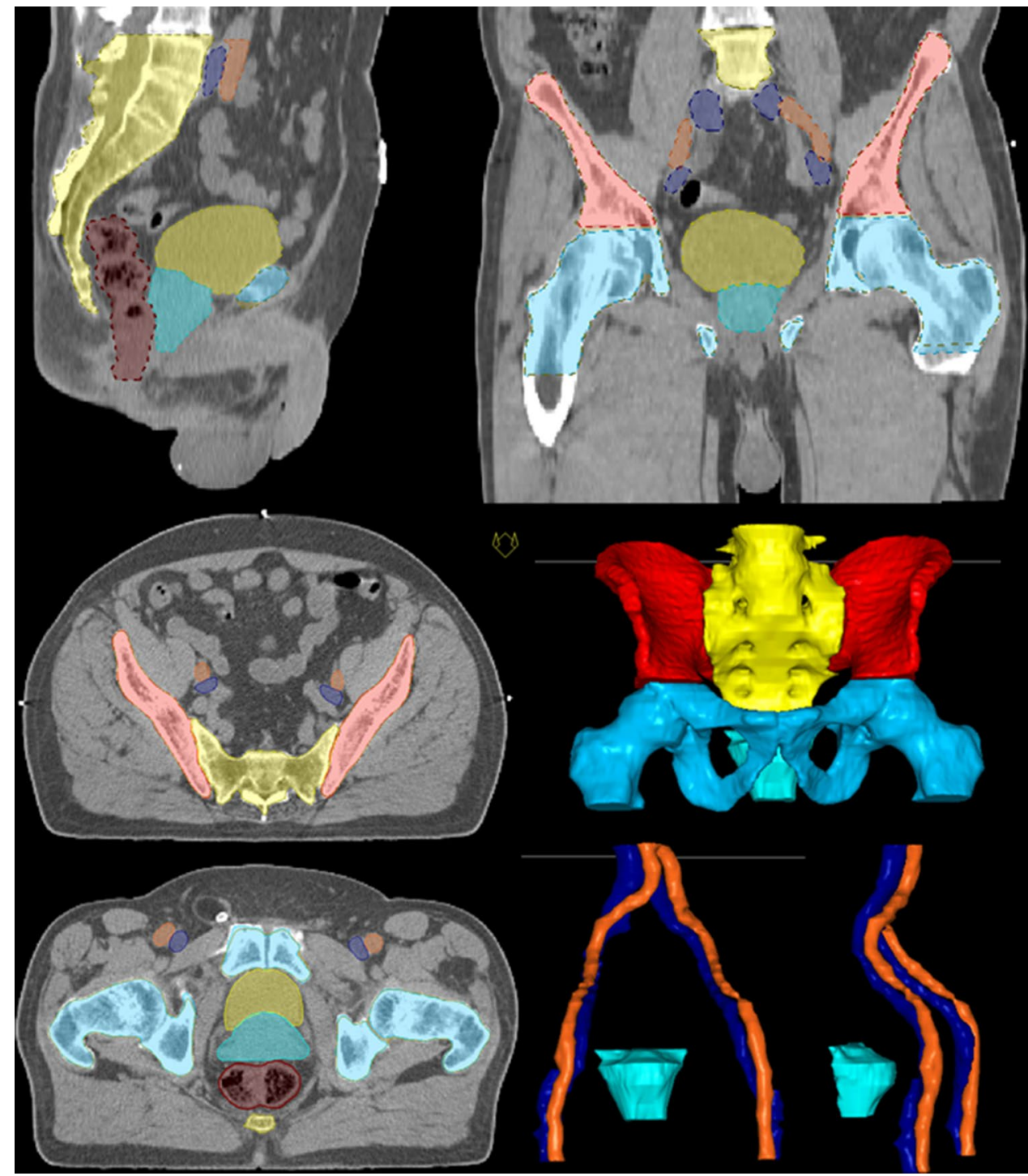

\section{Interdependence of CTV, V20 (PBM union) and V20 (vessels)}

In order to select parameters for comparison with immune cell subset changes, the interdependence of CTV, V20 (PBM union) and V20 (vessels) was determined. Whereas a Pearson correlation coefficient of 0.61 indicates a moderate correlation between V20 (vessels) and V20 (PBM union) (Fig. 4, lower panel), V20 (vessels) and V20 (PBM union) showed no or only moderate correlations with the CTV (Fig. 4, second row panels). Thus, all three parameters were considered for subsequent analysis in conjunction with immune cell changes over the course of treatment. Most strong or moderate correlations with radiotherapy planning parameters were found at the early time point 4 weeks into radiotherapy $(\mathrm{B} / \mathrm{A})$, while only one moderate correlation occurred at the end of radiotherapy (data not shown).

\section{Correlation between $\mathrm{T}$ cell ratios and radiotherapy planning volumes}

The $\mathrm{T}$ cell ratios showed a moderate negative correlation with CTV volume for time points $\mathrm{B} / \mathrm{A}$ and $\mathrm{D} / \mathrm{A}$, while no correlation was found for time points C/A (Fig. 5, upper panel). Ratio C/A was the only time point with a significant decrease in $\mathrm{T}$ cells compared to baseline (Fig. 1). $\mathrm{T}$ cell proliferation showed a moderate positive correlation with CTV volume for ratio D/A only (Fig. 5, lower panel), while the absolute values of $\mathrm{T}$ cell proliferation were the lowest at this time point and did not differ significantly from baseline. For patients receiving pelvic nodal irradiation (data not shown), for which an exploratory analysis was conducted, $\mathrm{T}$ cell ratios differed to those of patients 
Fig. 4 Dose-volume histograms of PBM union and vessels (upper panels) are shown for two patients comparing radiotherapy to the prostate only and radiotherapy including pelvic node irradiation. Correlations of CTV and V20 of vessels and PBM are shown for all patients. The clinical target volume (CTV) used for radiation planning and dose parameters for PBM and vessels showed no to moderate correlation to each other. Thus, the volume of CTV is not an adequate surrogate parameter for the dose to PBM and vessels (V20). V20 for PBM and V20 for vessels showed a moderate correlation
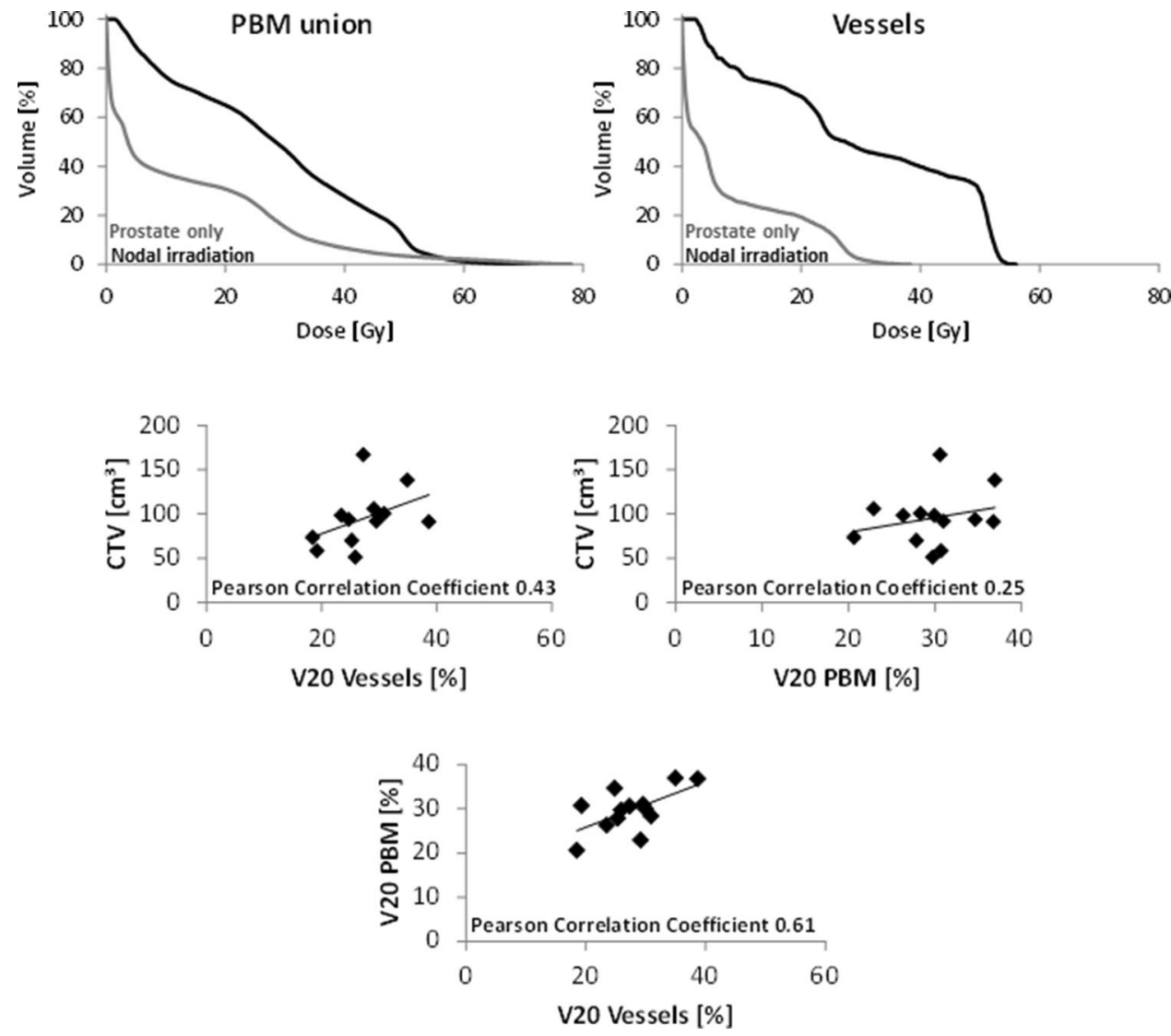

Ratio B/A

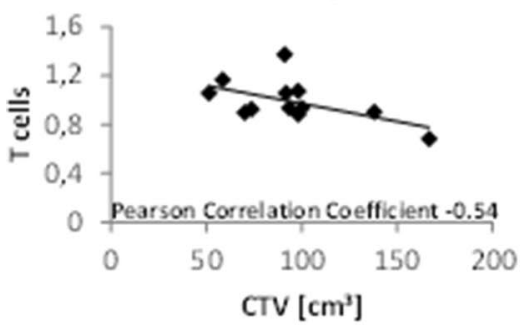

Ratio C/A
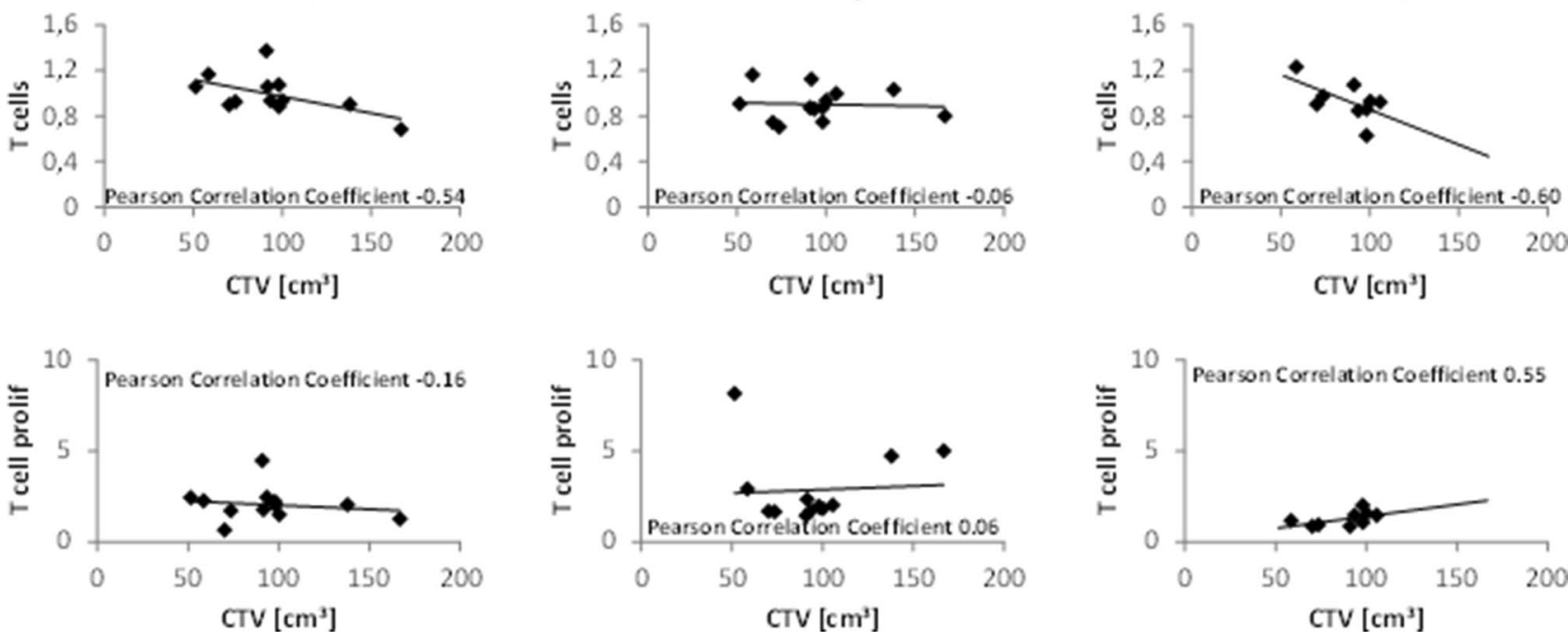

Fig. 5 Changes of $\mathrm{T}$ cells and $\mathrm{T}$ cell proliferation are shown for time points B/A, C/A and D/A in the primary analysis of patients receiving radiotherapy to prostate only. T cells negatively correlated with CTV volume when comparing time points $\mathrm{B}$ and $\mathrm{D}$, but not $\mathrm{C}$, to $\mathrm{A}$. T cell proliferation was positively correlated with CTV volume for ratio $\mathrm{D} / \mathrm{A}$, while having the lowest total ratio at this time point 
treated to the prostate only, showing a strong positive correlation with $\mathrm{CTV}$ volume for time point ratios $\mathrm{B} / \mathrm{A}$ and C/A (0.86 and 0.89, respectively). For ratio D/A, however, a moderate negative correlation was found $(-0.45)$. Again, $\mathrm{T}$ cell proliferation only showed a correlation for ratio $\mathrm{D} / \mathrm{A}$, although here, a moderate negative correlation with CTV volume could be observed (-0.52). These data do not confirm the results of the primary analysis.

\section{Correlation between $\mathrm{CD}^{+}$effector cells and Treg cells and radiotherapy planning volumes}

Early changes (B/A) in $\mathrm{CD}^{+}$effector cells, both not significantly different from baseline, showed a moderate negative correlation with V20 (vessels) as well as a strong negative correlation with V20 (PBM) (Pearson coefficients: - 0.66 and -0.73 , respectively). At the same time, Treg cell ratios showed moderate positive correlations with both volume parameters (Fig. 6, upper panels). No correlation was found at later time points at the end of radiotherapy or 3 months thereafter (Fig. 6, lower panels). An exploratory analysis of the five patients who underwent radiotherapy to the prostate as well as pelvic nodal regions hints at a confirmation of those results with positive correlations of V20 (vessels) and
V20 (PBM) with Treg cells (Pearson correlation coefficient 0.80 and $0.93,0.90$ and $0.94,0.87$ and 0.74 for ratios B/A, $\mathrm{C} / \mathrm{A}$ and D/A, respectively). The negative correlation of V20 (vessels) and V20 (PBM) with $\mathrm{CD}^{+}$effector cells was confirmed as well for time points during treatment (Pearson correlation coefficient -0.74 and $-0.99,-0.70$ and $-0.84,-0.42$ and -0.30 for ratios $\mathrm{B} / \mathrm{A}, \mathrm{C} / \mathrm{A}$ and $\mathrm{D} / \mathrm{A}$, respectively, data not shown).

\section{Discussion}

We demonstrated previously that peripheral immune cell subsets are affected during and after curative, normofractionated radiotherapy for intermediate to high-risk prostate cancer [21]. Here, we show that these changes are associated with patient age, target volume and especially with contoured immune-related volumes (bone marrow and blood vessels). These correlations are mostly observed early during treatment (4 weeks into radiotherapy as compared to levels before start of the treatment), and persist 3 months after end of treatment.

This small study included patients with localized, nodenegative prostate cancer. Especially for patients treated with
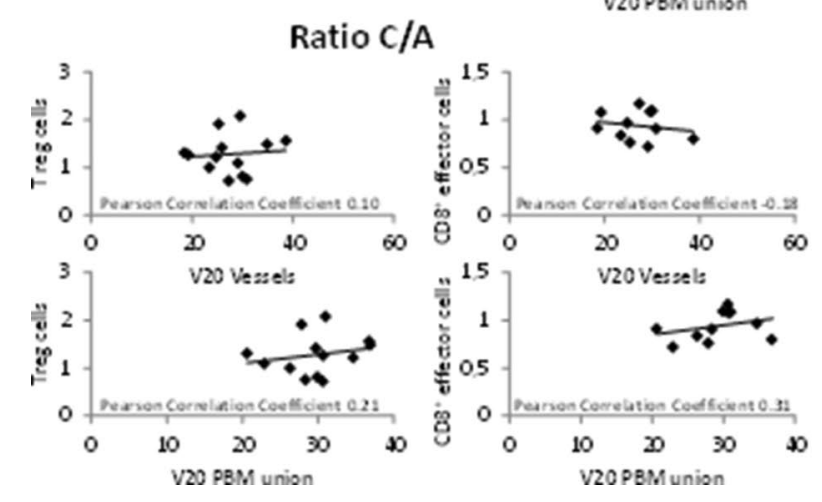

Fig. 6 Ratios of Treg cells as well as $\mathrm{CD}^{+}$effector cells are shown in correlation with V20 (blood vessels) and V20 (PBM union) for time points B/A, C/A and D/A. Treg cells correlated positively with V20 (blood vessels) as well as V20 (PBM) at time point B/A in the

\section{Ratio B/A}
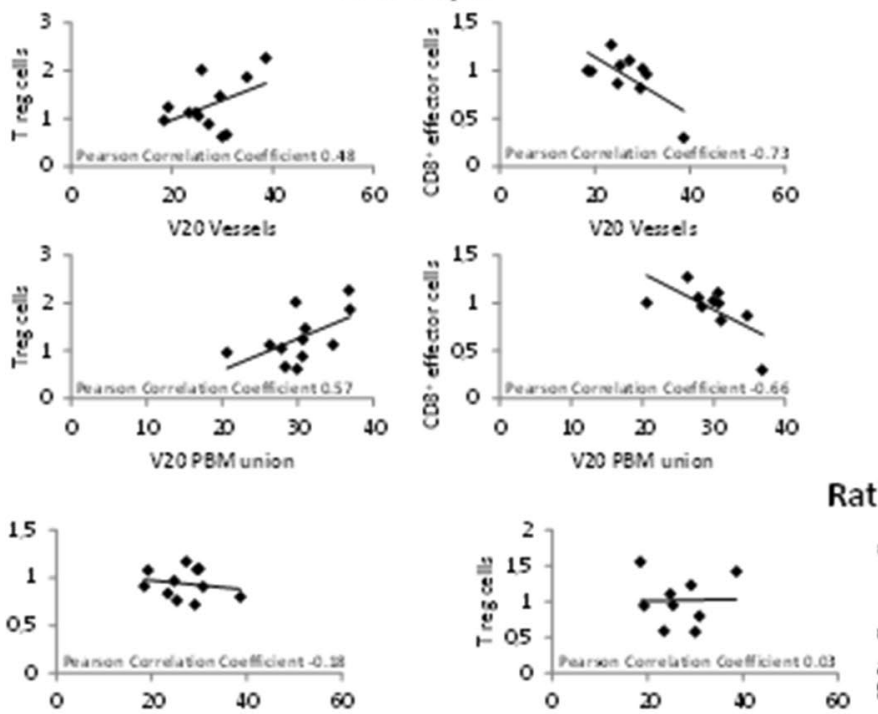

Ratio D/A
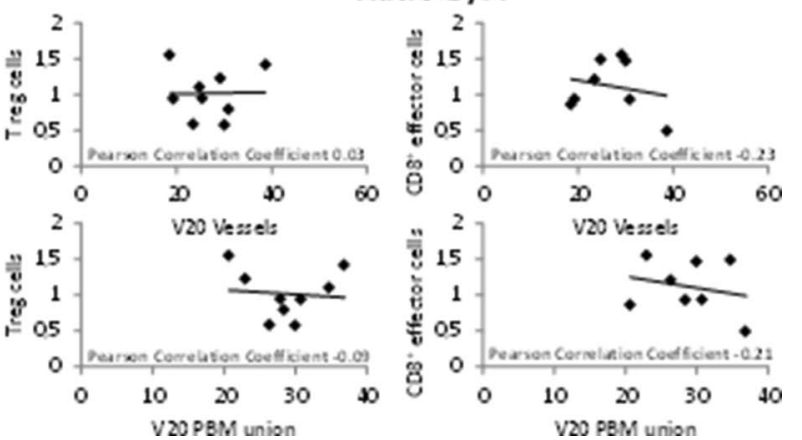

primary analysis. At the same time, $\mathrm{CD}^{+}$effector cell ratios showed a negative correlation with V20 (blood vessels) as well as V20 (PBM). No such correlation was found with the ratios at time points $\mathrm{C} / \mathrm{A}$ or $\mathrm{D} / \mathrm{A}$ 
elective nodal radiation, no conclusions can be drawn from our data as the sample size is limited to five patients. In addition, clinical variables such as T stage and PSA as well as treatment concepts differed significantly. For the performed analysis, patients treated with radiotherapy to prostate with or without seminal vesicles were included in the primary analysis. Patients treated with additional elective nodal irradiation were analyzed separately in an exploratory analysis. Two patients with intermediate-risk prostate cancer declined the use of ADT in addition to radiotherapy, which might influence the immune status of the patients [33]. However, these patients were included in the primary analysis. CTV volume and thus possibly dose to immune-related volume might differ for patients with T3b tumors with inclusion of the whole seminal vesicles into the CTV.

It is well established that aging is associated with alterations of the immune system, especially in the T cell compartment. Frequencies of naïve $\mathrm{CD} 4^{+}$and $\mathrm{CD}^{+}{ }^{+} \mathrm{T}$ cells decrease with age in healthy individuals, while effector and memory subsets increase. In addition, $\mathrm{T}$ cell function is often impaired, in particular, in CMV positive individuals [34]. Such changes are thought to have an essential impact on prophylactic vaccination, but also on therapeutic manipulations of the immune system. As prostate cancer is mostly a disease of the elderly, these considerations are substantial. In animal models, $T$ cells from young mice are more effective at eliminating tumors than these of older mice [35]. In head and neck squamous cell xenografts, tumor growth was also shown to be more rapid in old mice compared to young ones. However, upon checkpoint inhibition, tumors of older mice responded better, and this was associated with higher expression levels of immune checkpoint molecules CTLA-4 and PD- 1 on T cells [36]. Similar observations have been made in some reports on melanoma patients treated with immune checkpoint inhibition [37].

The contouring strategy for pelvic bone marrow described by $\mathrm{Li}$ et al. [31] was confirmed. A significant difference between bone marrow and bone delineation was not found. Bone delineation is possible with semi-automatic contouring on CT only datasets such as planning CTs for radiotherapy [38]. In addition, since circulating lymphocytes have been described as being a "moving organ at risk" for radiotherapy $[39,40]$, large pelvic vessels were contoured. It has been recently proposed that immunotherapy combined to "lymphocyte sparing radiotherapy" could lead to improved clinical responses [41]. Vessel and bone marrow volumes were only partly associated with each other and with the volume of the CTV in our prostate carcinoma patient cohort, so that all three parameters were used for further analysis.

The volume of the CTV was inversely associated with changes of total $\mathrm{T}$ cells early during radiotherapy, as well as in the recovery phase after end of treatment. Sage et al. described a decrease in $\mathrm{T}$ cells at the end of radiotherapy for prostate cancer patients. However, they did not investigate associations with CTV volumes or other radiotherapy planning parameters [42].

During radiotherapy, large volumes of bone marrow and vessels receiving radiation doses $>20$ Gy were associated with higher ratios of Treg cells and lower ratios of $\mathrm{CD} 8^{+}$ effector cells. These data suggest an early impairment of anti-tumor immunity with larger irradiated immune-related volumes and go along with the known radioresistance of Tregs. Treg cells are major immunosuppressive players in the context of tumors, and normofractionated radiotherapy has been described to lead to an accumulation of Treg cells [43]. Immunohistochemical staining of the Treg cell marker FoxP3 following neoadjuvant radiochemotherapy for esophageal cancer has been reported as a prognostic marker for cancer-specific survival [44]. In contrast, CD8 ${ }^{+}$ effector cells are the main effectors of anti-tumor immune response (reviewed in [45]). For instance, in patients receiving stereotactic radiotherapy for oligometastatic prostate cancer, an increase in tumor-reactive $\mathrm{CD} 8^{+}$effector cells was correlated with local disease control [46]. Whereas irradiation can promote $\mathrm{T}$ cell activation via dendritic cells in preclinical tumor models in vivo [47], patients treated with radiochemotherapy for cervical cancer showed significant reduction of $\mathrm{CD}^{+} \mathrm{T}$ cells during treatment [19]. Cytotoxic $\mathrm{T}$ cells have been linked to the outcome of patients with several cancers, e.g., anal cancer treated with radiochemotherapy [48]. Combinatorial approaches of radiotherapy and immunotherapy seem to be dependent on activation of $\mathrm{CD}^{+}$effector cells via dendritic cells [12]. Thus, the increase in Tregs and decrease in $\mathrm{CD}^{+}$effector cells with larger volumes of immune-related structures receiving radiation doses $>20$ Gy at an early time point during treatment might reflect a transient impairment of the anti-tumor immunity. Irradiation of large volumes, especially elective nodal regions, has received a lot of attention concerning possible negative effects on anti-cancer immunity, so new concepts might emerge for node positive prostate cancer, especially in settings combining radiotherapy with immunotherapy [49]. These hypotheses might also form the basis on limiting the target volume margins in prostate radiotherapy for deliberate sparing of the obturator region.

Pelvic bone marrow volumes were already used to generate dosimetric data associated with acute hematologic toxicity during radio(chemo)therapy of pelvic malignancies, mostly rectal or anal cancer [50-55]. Those findings have led to the concept of bone marrow sparing radiotherapy to avoid these toxicities [52, 56, 57]. However, experimental studies did not differentiate immune cell subsets in comparable depth to our study. In addition, sparing of bone marrow might lead to higher radiation doses in other immune-related pelvic volumes. 


\section{Conclusions}

In conclusion, especially early changes of peripheral immune status during radiotherapy as well as long-lasting changes during the recovery phase appear to correlate with radiotherapy planning volumes and irradiated volumes of immune-associated pelvine structures in definitive treatment of prostate cancer. Maximal changes at the end of treatment seem to be mostly independent of radiotherapy planning parameters in this clinical setting. In order to further characterize the dependence of immune changes during treatment with radiotherapy planning parameters, early and late time points should be included in future analyses. Early impairment of anti-tumor immunity with larger irradiated volumes might provide a rationale for actively sparing immune-related volumes during prostate cancer radiotherapy, especially in combinatorial approaches with immunotherapy strategies.

Supplementary Information The online version contains supplementary material available at https://doi.org/10.1007/s00262-021-03002-6.

Funding Open Access funding enabled and organized by Projekt DEAL. This work was supported by the Medical Faculty of Tuebingen under Grant 261-0-0. E. Hoffmann: Medical Faculty Tuebingen (Junior Clinician Scientist Program, Grant 444-0-0). F. Paulsen: Funding: None. P. Schaedle: Funding: None. D. Zips: Funding: None. C. Gani: Funding: None. H.-G. Rammensee: Cluster of Excellence iFIT (EXC2180) "Image-Guided and Functionally Instructed Tumor Therapies", German Cancer Consortium (DKTK) and German Cancer Research Center (DKFZ) partner site Tübingen. C. Gouttefangeas: Cluster of Excellence iFIT (EXC2180) "Image-Guided and Functionally Instructed Tumor Therapies," German Cancer Consortium (DKTK) and German Cancer Research Center (DKFZ) partner site Tübingen. F. Eckert: Medical Faculty Tuebingen (AKF grant 261-0-0, PATE grant 2007-0-0, TUEFF grant 2154- 0-0), Research Fellowship by the Else-Kroener-Fresenius Foundation, German Cancer Aid grant 70114187.

Data availability Research data will be shared upon request.

\section{Declarations}

Conflicts of interest E. Hoffmann: Research and educational grants from Elekta, Philips, Siemens, and Sennewald. F. Paulsen: Research and educational grants from Elekta, Philips, Siemens, and Sennewald. P. Schaedle: No conflict of interest. D. Zips: Research and educational grants by Elekta, Philips, Siemens, Sennewald, Kaikuu, and TheraPanacea C. Gani: Research and educational grants, sponsoring for symposia by Elekta, Philips, Siemens, Sennewald, Kaikuu, and TheraPanacea. H.-G. Rammensee: No conflict of interest. C. Gouttefangeas: No conflict of interest. F. Eckert: Research and educational grants from Elekta, Philips, Siemens, and Sennewald. Speaker's honoraria by Sennewald.

Ethical approval The study was approved by the local ethics committee (project number 402/2010BO2). All patients had signed informed consent.
Open Access This article is licensed under a Creative Commons Attribution 4.0 International License, which permits use, sharing, adaptation, distribution and reproduction in any medium or format, as long as you give appropriate credit to the original author(s) and the source, provide a link to the Creative Commons licence, and indicate if changes were made. The images or other third party material in this article are included in the article's Creative Commons licence, unless indicated otherwise in a credit line to the material. If material is not included in the article's Creative Commons licence and your intended use is not permitted by statutory regulation or exceeds the permitted use, you will need to obtain permission directly from the copyright holder. To view a copy of this licence, visit http://creativecommons.org/licenses/by/4.0/.

\section{References}

1. Viani GA, Stefano EJ, Afonso SL (2009) Higher-than-conventional radiation doses in localized prostate cancer treatment: a meta-analysis of randomized, controlled trials. Int J Radiat Oncol Biol Phys 74:1405-1418. https://doi.org/10.1016/j.ijrobp. 2008.10.091

2. Podder TK, Fredman ET, Ellis RJ (2018) Advances in radiotherapy for prostate cancer treatment. Adv Exp Med Biol 1096:31-47. https://doi.org/10.1007/978-3-319-99286-0_2

3. Mohler JL, Antonarakis ES, Armstrong AJ et al (2019) Prostate cancer, version 2.2019, NCCN clinical practice guidelines in oncology. J Natl Compr Canc Netw 17:479-505. https://doi. org/10.6004/jnccn.2019.0023

4. Yang F, Markovic SN, Molina JR et al (2020) Association of sex, age, and eastern cooperative oncology group performance status with survival benefit of cancer immunotherapy in randomized clinical trials: a systematic review and meta-analysis. JAMA Netw Open 3:e2012534. https://doi.org/10.1001/jaman etworkopen.2020.12534

5. Kim TJ, Koo KC (2020) Current status and future perspectives of checkpoint inhibitor immunotherapy for prostate cancer: a comprehensive review. Int J Mol Sci. https://doi.org/10.3390/ ijms21155484

6. Comiskey MC, Dallos MC, Drake CG (2018) Immunotherapy in prostate cancer: teaching an old dog new tricks. Curr Oncol Rep 20:75. https://doi.org/10.1007/s11912-018-0712-z

7. Feyerabend S, Stevanovic S, Gouttefangeas C et al (2009) Novel multi-peptide vaccination in Hla-A2+ hormone sensitive patients with biochemical relapse of prostate cancer. Prostate 69:917-927. https://doi.org/10.1002/pros.20941

8. Grassberger C, Ellsworth SG, Wilks MQ, Keane FK, Loeffler JS (2019) Assessing the interactions between radiotherapy and antitumour immunity. Nat Rev Clin Oncol 16:729-745. https:// doi.org/10.1038/s41571-019-0238-9

9. Reynders K, De Ruysscher D (2015) Radiotherapy and immunotherapy: improving cancer treatment through synergy. Prog Tumor Res 42:67-78. https://doi.org/10.1159/000437185

10. Wang Y, Liu ZG, Yuan H, Deng W, Li J, Huang Y, Kim BYS, Story MD, Jiang W (2019) The Reciprocity between radiotherapy and cancer immunotherapy. Clin Cancer Res 25:1709-1717. https://doi.org/10.1158/1078-0432.Ccr-18-2581

11. Deutsch E, Chargari C, Galluzzi L, Kroemer G (2019) Optimising efficacy and reducing toxicity of anticancer radioimmunotherapy. Lancet Oncol 20:e452-e463. https://doi.org/10.1016/ s1470-2045(19)30171-8

12. Dovedi SJ, Lipowska-Bhalla G, Beers SA, Cheadle EJ, Mu L, Glennie MJ, Illidge TM, Honeychurch J (2016) Antitumor efficacy of radiation plus immunotherapy depends upon dendritic cell activation of effector CD8+ T Cells. Cancer Immunol Res 4:621-630. https://doi.org/10.1158/2326-6066.Cir-15-0253 
13. Harris TJ, Hipkiss EL, Borzillary S et al (2008) Radiotherapy augments the immune response to prostate cancer in a timedependent manner. Prostate 68:1319-1329. https://doi.org/10. 1002/pros.20794

14. Vanpouille-Box C, Alard A, Aryankalayil MJ et al (2017) DNA exonuclease Trex 1 regulates radiotherapy-induced tumour immunogenicity. Nat Commun 8:15618. https://doi.org/10. 1038/ncomms 15618

15. Eckert F, Gaipl US, Niedermann G, Hettich M, Schilbach K, Huber SM, Zips D (2017) Beyond checkpoint inhibition Immunotherapeutical strategies in combination with radiation. Clin Transl Radiat Oncol 2:29-35. https://doi.org/10.1016/j. ctro.2016.12.006

16. Eckert F, Zwirner K, Boeke S, Thorwarth D, Zips D, Huber SM (2019) Rationale for combining radiotherapy and immune checkpoint inhibition for patients with hypoxic tumors. Front Immunol 10:407. https://doi.org/10.3389/fimmu.2019.00407

17. Donaubauer AJ, Rühle PF, Becker I, Fietkau R, Gaipl US, Frey B (2019) One-tube multicolor flow cytometry assay (OTMA) for comprehensive immunophenotyping of peripheral blood. Methods Mol Biol 1904:189-212. https://doi.org/10.1007/ 978-1-4939-8958-4_8

18. Neo SY, O'Reilly A, Pico de Coaña Y (2019) Immune monitoring of cancer patients by multi-color flow cytometry. Methods Mol Biol 1913:49-65. https://doi.org/10.1007/978-1-4939-8979-9_4

19. van Meir H, Nout RA, Welters MJ et al (2016) Impact of (chemo) radiotherapy on immune cell composition and function in cervical cancer patients. Oncoimmunology. 6:e1267095

20. Riemann D, Cwikowski M, Turzer S, Giese T, Grallert M, Schütte W, Seliger B (2019) Blood immune cell biomarkers in lung cancer. Clin Exp Immunol 195:179-189. https://doi.org/10.1111/cei. 13219

21. Eckert F, Schaedle P, Zips D, Schmid-Horch B, Rammensee HG, Gani C, Gouttefangeas C (2018) Impact of curative radiotherapy on the immune status of patients with localized prostate cancer. Oncoimmunology 7:e1496881. https://doi.org/10.1080/2162402x. 2018.1496881

22. Yan K, Ramirez E, Xie XJ, Gu X, Xi Y, Albuquerque K (2018) Predicting severe hematologic toxicity from extended-field chemoradiation of para-aortic nodal metastases from cervical cancer. Pract Radiat Oncol 8:13-19. https://doi.org/10.1016/j.prro.2017. 07.001

23. Bazan JG, Luxton G, Mok EC, Koong AC, Chang DT (2012) Normal tissue complication probability modeling of acute hematologic toxicity in patients treated with intensity-modulated radiation therapy for squamous cell carcinoma of the anal canal. Int J Radiat Oncol Biol Phys 84:700-706. https://doi.org/10.1016/j. ijrobp.2011.12.072

24. Cozzarini C, Noris Chiorda B, Sini C, Fiorino C, Briganti A, Montorsi F, Di Muzio N (2016) Hematologic toxicity in patients treated with postprostatectomy whole-pelvis irradiation with different intensity modulated radiation therapy techniques is not negligible and is prolonged: preliminary results of a longitudinal, observational study. Int J Radiat Oncol Biol Phys 95:690-695. https://doi.org/10.1016/j.ijrobp.2016.01.022

25. Sini C, Fiorino C, Perna L et al (2016) Dose-volume effects for pelvic bone marrow in predicting hematological toxicity in prostate cancer radiotherapy with pelvic node irradiation. Radiother Oncol 118:79-84. https://doi.org/10.1016/j.radonc.2015.11.020

26. Farhood B, Najafi M, Mortezaee K (2019) CD8(+) cytotoxic T lymphocytes in cancer immunotherapy: a review. J Cell Physiol 234:8509-8521. https://doi.org/10.1002/jcp.27782

27. Reading JL, Gálvez-Cancino F, Swanton C, Lladser A, Peggs KS, Quezada SA (2018) The function and dysfunction of memory CD8(+) T cells in tumor immunity. Immunol Rev 283:194-212. https://doi.org/10.1111/imr.12657
28. Tanaka A, Sakaguchi S (2017) Regulatory T cells in cancer immunotherapy. Cell Res 27:109-118. https://doi.org/10.1038/cr.2016. 151

29. Baum C, Alber M, Birkner M, Nusslin F (2006) Robust treatment planning for intensity modulated radiotherapy of prostate cancer based on coverage probabilities. Radiother Oncol 78:27-35. https://doi.org/10.1016/j.radonc.2005.09.005

30. Eckert F, Alloussi S, Paulsen F et al (2013) Prospective evaluation of a hydrogel spacer for rectal separation in dose-escalated intensity-modulated radiotherapy for clinically localized prostate cancer. BMC Cancer 13:27. https://doi.org/10.1186/1471-2407-13-27

31. Li N, Liu X, Zhai F, Liu B, Cao X, Li S, Zhang M, Liu M (2017) Association between dose-volume parameters and acute bone marrow suppression in rectal cancer patients treated with concurrent chemoradiotherapy. Oncotarget 8:92904-92913. https:// doi.org/10.18632/oncotarget.21646

32. Nakamura N, Kusunoki Y, Akiyama M (1990) Radiosensitivity of CD4 or CD8 positive human T-lymphocytes by an in vitro colony formation assay. Radiat Res 123:224-227

33. Kalina JL, Neilson DS, Comber AP, Rauw JM, Alexander AS, Vergidis J, Lum JJ (2017) Immune modulation by androgen deprivation and radiation therapy: implications for prostate cancer immunotherapy. Cancers. https://doi.org/10.3390/cancers9020013

34. Fülöp T, Larbi A, Pawelec G (2013) Human T cell aging and the impact of persistent viral infections. Front Immunol 4:271. https:// doi.org/10.3389/fimmu.2013.00271

35. Schreiber K, Arina A, Engels B et al (2012) Spleen cells from young but not old immunized mice eradicate large established cancers. Clin Cancer Res 18:2526-2533. https://doi.org/10.1158/ 1078-0432.Ccr-12-0127

36. Sekido K, Tomihara K, Tachinami H, Heshiki W, Sakurai K, Moniruzzaman R, Imaue S, Fujiwara K, Noguchi M (2019) Alterations in composition of immune cells and impairment of anti-tumor immune response in aged oral cancer-bearing mice. Oral Oncol 99:104462. https://doi.org/10.1016/j.oraloncology. 2019.104462

37. Kugel CH 3rd, Douglass SM, Webster MR et al (2018) Age Correlates with Response to Anti-PD1, Reflecting Age-Related Differences in Intratumoral Effector and Regulatory T-Cell Populations. Clin Cancer Res 24:5347-5356. https://doi.org/10.1158/ 1078-0432.Ccr-18-1116

38. Wang S, Nie D, Qu L, Shao Y, Lian J, Wang Q, Shen D (2020) CT Male pelvic organ segmentation via hybrid loss network with incomplete annotation. IEEE Trans Med Imaging 39:2151-2162. https://doi.org/10.1109/tmi.2020.2966389

39. Jin JY, Mereniuk T, Yalamanchali A, Wang W, Machtay M, Spring Kong FM, Ellsworth S (2020) A framework for modeling radiation induced lymphopenia in radiotherapy. Radiother Oncol 144:105-113. https://doi.org/10.1016/j.radonc.2019.11.014

40. Wang X, Wang P, Zhao Z, Mao Q, Yu J, Li M (2020) A review of radiation-induced lymphopenia in patients with esophageal cancer: an immunological perspective for radiotherapy. Ther Adv Med Oncol 12:1758835920926822. https://doi.org/10.1177/17588 35920926822

41. Lambin P, Lieverse RIY, Eckert F, Marcus D, Oberije C, van der Wiel AMA, Guha C, Dubois LJ, Deasy JO (2020) Lymphocytesparing radiotherapy: the rationale for protecting lymphocyte-rich organs when combining radiotherapy with immunotherapy. Semin Radiat Oncol 30:187-193. https://doi.org/10.1016/j.semradonc. 2019.12.003

42. Sage EK, Schmid TE, Geinitz H, Gehrmann M, Sedelmayr M, Duma MN, Combs SE, Multhoff G (2017) Effects of definitive and salvage radiotherapy on the distribution of lymphocyte subpopulations in prostate cancer patients. Strahlenther Onkol 193:648-655. https://doi.org/10.1007/s00066-017-1144-7 
43. Verma A, Mathur R, Farooque A, Kaul V, Gupta S, Dwarakanath BS (2019) T-Regulatory Cells In Tumor Progression And Therapy. Cancer Manag Res 11:10731-10747. https://doi.org/10.2147/ cmar.S228887

44. Vacchelli E, Semeraro M, Enot DP et al (2015) Negative prognostic impact of regulatory $\mathrm{T}$ cell infiltration in surgically resected esophageal cancer post-radiochemotherapy. Oncotarget 6:20840 20850. https://doi.org/10.18632/oncotarget.4428

45. Demaria S, Formenti SC (2012) Role of T lymphocytes in tumor response to radiotherapy. Front Oncol 2:95. https://doi.org/10. 3389/fonc.2012.00095

46. Evans JD, Morris LK, Zhang $\mathrm{H}$ et al (2019) Prospective immunophenotyping of CD8(+) t cells and associated clinical outcomes of patients with oligometastatic prostate cancer treated with metastasis-directed SBRT. Int J Radiat Oncol Biol Phys 103:229-240. https://doi.org/10.1016/j.ijrobp.2018.09.001

47. Gupta A, Probst HC, Vuong V et al (2012) Radiotherapy promotes tumor-specific effector CD8+ T cells via dendritic cell activation. Journal of immunology 189:558-66. https://doi.org/10.4049/ jimmunol.1200563

48. Grabenbauer GG, Lahmer G, Distel L, Niedobitek G (2006) Tumor-infiltrating cytotoxic $\mathrm{T}$ cells but not regulatory $\mathrm{T}$ cells predict outcome in anal squamous cell carcinoma. Clin Cancer Res 12:3355-3360. https://doi.org/10.1158/1078-0432.Ccr-05-2434

49. Friedrich T, Henthorn N, Durante M (2021) Modeling radioimmune response-current status and perspectives. Front Oncol 11:647272. https://doi.org/10.3389/fonc.2021.647272

50. Franco P, Ragona R, Arcadipane F, Mistrangelo M, Cassoni P, Rondi N, Morino M, Racca P, Ricardi U (2016) Lumbar-sacral bone marrow dose modeling for acute hematological toxicity in anal cancer patients treated with concurrent chemo-radiation. Med Oncol 33:137. https://doi.org/10.1007/s12032-016-0852-7

51. Franco P, Ragona R, Arcadipane F, Mistrangelo M, Cassoni P, Rondi N, Morino M, Racca P, Ricardi U (2017) Dosimetric predictors of acute hematologic toxicity during concurrent intensity-modulated radiotherapy and chemotherapy for anal cancer. Clin Transl Oncol 19:67-75. https://doi.org/10.1007/ s12094-016-1504-2
52. Jianyang W, Yuan T, Yuan T et al (2016) A prospective phase II study of magnetic resonance imaging guided hematopoietical bone marrow-sparing intensity-modulated radiotherapy with concurrent chemotherapy for rectal cancer. Radiol Med 121:308-314. https://doi.org/10.1007/s11547-015-0605-2

53. Kumar T, Schernberg A, Busato F, Laurans M, Fumagalli I, Dumas I, Deutsch E, Haie-Meder C, Chargari C (2019) Correlation between pelvic bone marrow radiation dose and acute hematological toxicity in cervical cancer patients treated with concurrent chemoradiation. Cancer Manag Res 11:6285-6297. https:// doi.org/10.2147/cmar.S195989

54. Mell LK, Schomas DA, Salama JK et al (2008) Association between bone marrow dosimetric parameters and acute hematologic toxicity in anal cancer patients treated with concurrent chemotherapy and intensity-modulated radiotherapy. Int J Radiat Oncol Biol Phys 70:1431-1437. https://doi.org/10.1016/j.ijrobp. 2007.08.074

55. Rose B, Mitra D, Hong TS et al (2017) Irradiation of anatomically defined pelvic subsites and acute hematologic toxicity in anal cancer patients undergoing chemoradiation. Pract Radiat Oncol 7:e291-e297. https://doi.org/10.1016/j.prro.2017.03.008

56. Mell LK, Tiryaki H, Ahn KH, Mundt AJ, Roeske JC, Aydogan B (2008) Dosimetric comparison of bone marrow-sparing intensitymodulated radiotherapy versus conventional techniques for treatment of cervical cancer. Int J Radiat Oncol Biol Phys 71:15041510. https://doi.org/10.1016/j.ijrobp.2008.04.046

57. Platta CS, Bayliss A, McHaffie D, Tome WA, Straub MR, Bradley KA (2013) A dosimetric analysis of tomotherapy based intensity modulated radiation therapy with and without bone marrow sparing in gynecologic malignancies. Technol Cancer Res Treat 12:19-29. https://doi.org/10.7785/tcrt.2012.500300

Publisher's Note Springer Nature remains neutral with regard to jurisdictional claims in published maps and institutional affiliations. 